\title{
molecules
}

ISSN 1420-3049

www.mdpi.com/journal/molecules

Article

\section{Synthesis and Bioactivity of Pyrazole Acyl Thiourea Derivatives}

\section{Jian Wu ${ }^{\dagger}$, Qing Shi ${ }^{\dagger}$, Zhuo Chen, Ming He, Linhong Jin and Deyu Hu *}

State Key Laboratory Breeding Base of Green Pesticide and Agricultural Bioengineering, Key Laboratory of Green Pesticide and Agriculture Bioengineering, Ministry of Education, Guizhou University, Guiyang 550025, China; E-Mails: jianwu2691@yahoo.com.cn (J.W.); shiqing982816@163.com (Q.S.); fcc.zchen@gzu.edu.cn (Z.C.); hmher@126.com (M.H.); fcc.jinlh@gzu.edu.cn (L.J.)

$\dagger$ These authors contributed equally to this work.

* Author to whom correspondence should be addressed; E-Mail: fcc.dyhu@gzu.edu.cn; Tel./Fax: +86-851-829-2170.

Received: 11 April 2012; in revised form: 19 April 2012 / Accepted: 19 April 2012 /

Published: 3 May 2012

\begin{abstract}
Sixteen novel pyrazole acyl thiourea derivatives 6 were synthesized from monomethylhydrazine (phenylhydrazine) and ethyl acetoacetate. The key 5-chloro-3methyl-1-substituted-1H-pyrazole-4-carbonyl chloride intermediates 4 were first generated in four steps through cyclization, formylation, oxidation and acylation. Thess were then reacted with ammonium thiocyanate in the presence of PEG-400 to afford 5-chloro-3methyl-1-substituted-1H-pyrazole-4-carbonyl isothiocyanates 5. Subsequent reaction with fluorinated aromatic amines resulted in the formation of the title compounds. The synthesized compound were unequivocally characterized by IR, ${ }^{1} \mathrm{H}-\mathrm{NMR},{ }^{13} \mathrm{C}-\mathrm{NMR}$ and elemental analysis and some of the synthesized compounds displayed good antifungal activities against Gibberella zeae, Fusarium oxysporum, Cytospora mandshurica and anti-TMV activity in preliminary antifungal activity tests.
\end{abstract}

Keywords: pyrazole acyl thiourea derivatives; synthesis; antifungal activity; antiviral activity

\section{Introduction}

Many acyl thiourea derivatives are well known to possess a wide range of bioactivities and are often employed as fungicidal, antiviral and regulating activities for plant protection in agriculture [1-3]. 
Some of them are employed as commercial insecticides [4], herbicides [5,6] and fungicides [7,8]. Several novel $N$ '-(substituted pyrimidin-2-yl)- $N$-chrysanthemoylthiourea derivatives were synthesized by Xu's group in 2005, and preliminary biological indicated that compounds I (Figure 1) exhibited $90 \%$ and $80 \%$ activity on Bipolaris maydis and Powdery mildew at $100 \mu \mathrm{g} / \mathrm{mL}$, respectively [9]. Compounds II and III (Figure 1), arylthioureas containing 1,3,4-thiadiazole moieties, were found to show good plant growth regulator activity at $10 \mu \mathrm{g} / \mathrm{mL}$ [10,11]; Wang et al. [12] reported that acyl thiourea derivatives IV exhibited excellent selective herbicidal activity towards dicotyledons. In addition, some novel $N$-[5-(2-chlorophenyl)-2-furamidothiocarbonyl]- $L$ - $\alpha$-amino acid ethyl esters (V) (see Figure 1) were found to show good activity against K562 cells in vitro at $10 \mu \mathrm{g} / \mathrm{mL}$ [13]. Moreover, compound VI (Figure 1) that can be prepared by a multi-step reaction sequence using 2-chloro-5-(chloromethyl) pyridine, displayed $96.4 \%$ and $85.3 \%$ activities against rape root and barnyard root at $10 \mu \mathrm{g} / \mathrm{mL}$, respectively [14].

Figure 1. The structure of acyl thiourea derivatives I-VI.

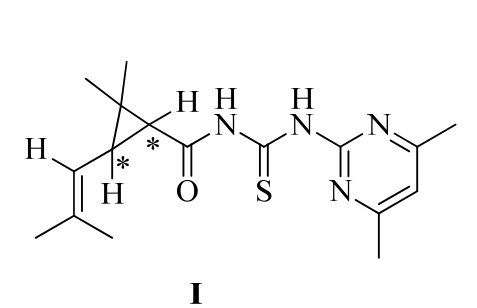<smiles>CC(Oc1ccccc1Cl)c1nnc(NC(=S)NC(=O)c2cccc(Cl)c2)s1</smiles><smiles>O=C(O)c1nnc(NC(=S)NC(=O)c2ccccc2Cl)s1</smiles>

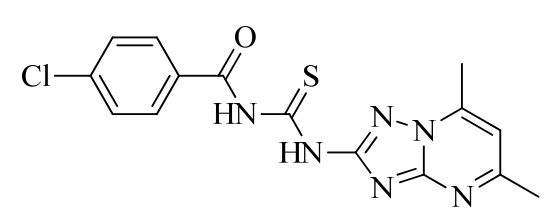

IV<smiles>[R]C(NC(=S)NC(=O)c1ccc(-c2ccccc2Cl)o1)C(=O)OCC</smiles>

$\mathbf{V}$<smiles>Cc1cc(C)nc(NC(=S)NC(=O)C2N=NN(Cc3ccc(Cl)nc3)C2C)n1</smiles>

VI

Pyrazole derivatives play an important role in the development of pesticides and medicines, which have become hot topics in recent years. Dozens of pyrazole products are available and widely used as fungicides, antiviral agents, analgesic agents, insecticides and herbicides [15-17]. In order to discover novel active compounds for use in agriculture, we sought to combine the active structures of thiourea and pyrazole to design and synthesize a class of novel pyrazole acyl thiourea derivatives. The synthetic route is shown in Scheme 1. All the compounds were unequivocally characterized by IR, NMR and elemental analysis. Preliminary bioassay tests indicated that most of the compounds exhibited antifungal activity against $G$. zeae, F. oxysporum and $C$. mandshurica and antiviral activity against TMV in vivo to a certain extent. Especially, compounds $\mathbf{6 b}, \mathbf{6 h}$ exhibited slightly superior or similar activity on the corresponding fungi as compared to the commercial hymexazol. 
Scheme 1. Synthetic route to compounds $\mathbf{6 a - p}$.

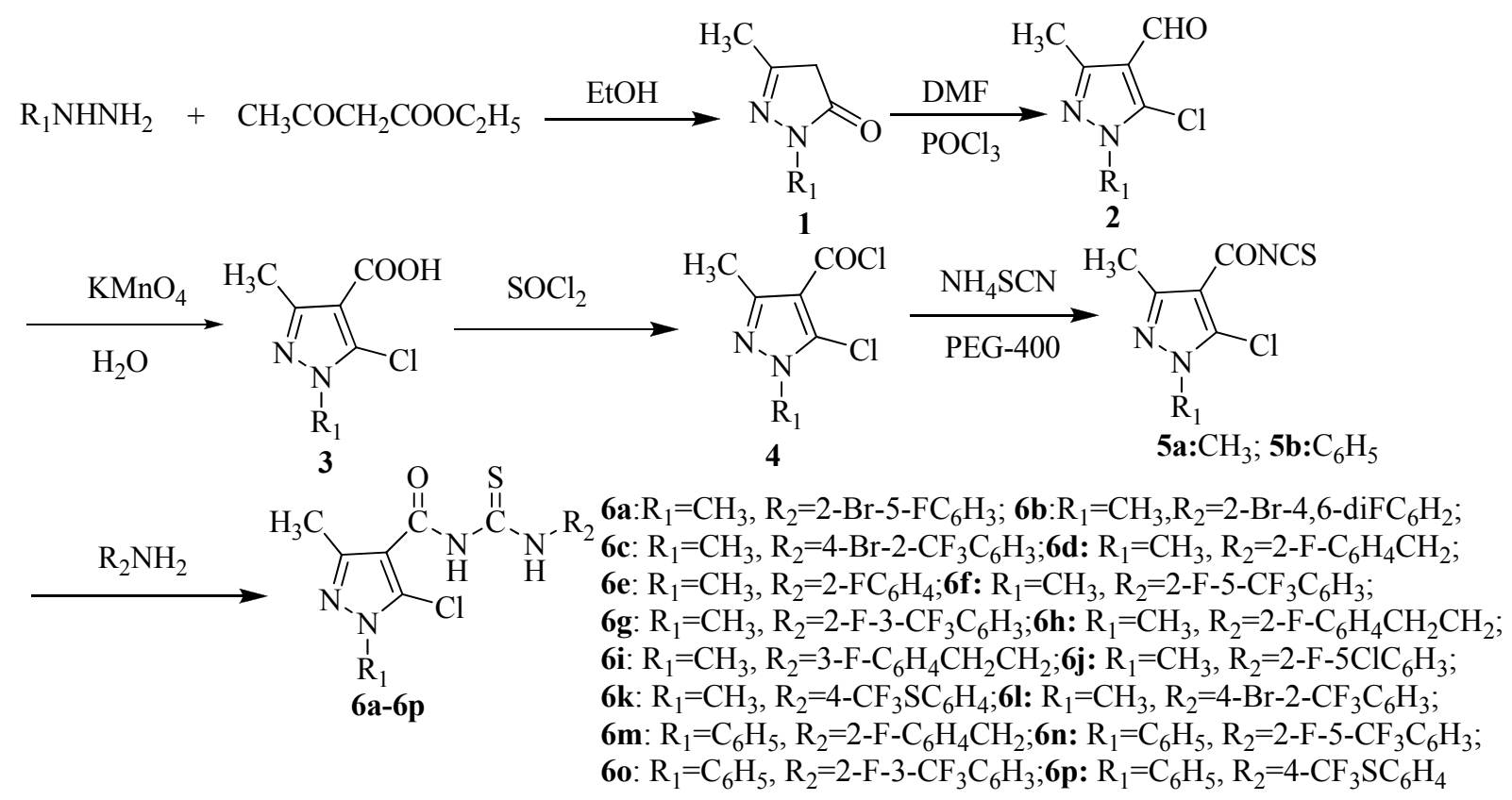

\section{Results and Discussion}

\subsection{Chemistry}

The synthetic route to title compounds $\mathbf{6 a}-\mathbf{p}$ is depicted in Scheme 1. In present study, we have conducted our reaction using PEG-400 as solid-liquid phase transfer catalyst (PTC). PEG-400 as a phase transfer catalyst is indispensable for these reactions; it can easily react with $\mathrm{NH}_{4} \mathrm{SCN}$ to form a [PEG-400-NH4 ${ }^{+}$] $\mathrm{SCN}^{-}$complex, which make it possible for $\mathrm{SCN}^{-}$to readily react with 5-chloro-1,3dimethyl-1H-pyrazole-4-carbonyl chloride and lead to the formation of intermediates 5a. In the preparation of 5-chloro-1,3-dimethyl-1H-pyrazole-4-carbonyl isothiocyanate 5a, phase transfer catalysis (PEG-400) and a conventional method were employed (see Table 1). From Table 1 we can see that the reaction in the presence of phase transfer catalysis (PEG-400) has many advantages such as low reaction temperatures and high yields. The use of PTC make the heterogeneous system react smoothly, the yield of the product be improved obviously $[18,19]$. The reaction of 5 with suitably substituted fluorinated primary aromatic amines afforded the desired acyl thioureas $\mathbf{6 a}-\mathbf{p}$ in good yields.

Table 1. Phase transfer catalysis compared with conventional method for $\mathbf{6 a}$.

\begin{tabular}{ccccc}
\hline Method & Agent & Reaction Temperature & Reaction Time & Yield \\
\hline phase transfer catalysis & $\mathrm{NH}_{4} \mathrm{SCN} / \mathrm{PEG}-400$ & $25^{\circ} \mathrm{C}$ & $3 \mathrm{~h}$ & $82.6 \%$ \\
conventional method & $\mathrm{NH}_{4} \mathrm{SCN}$ & $82^{\circ} \mathrm{C}$ & $3 \mathrm{~h}$ & $51.2 \%$ \\
\hline
\end{tabular}

The structures of the compounds $\mathbf{6 a}-\mathbf{p}$ were established on the basis of their spectroscopic data. The IR $(\mathrm{KBr})$ spectra of the target compounds $6 \mathbf{a}-\mathbf{p}$ showed characteristic $\mathrm{N}-\mathrm{H}, \mathrm{C}=\mathrm{O}$, and $\mathrm{C}=\mathrm{S}$ absorptions at 3,200-3,430 $\mathrm{cm}^{-1}, 1,660-1,690 \mathrm{~cm}^{-1}$ and $1,150 \mathrm{~cm}^{-1}$ respectively. The significantly reduced double bond character of the carbonyl group of the amide linkage accounts for the appearance 
of its stretching frequency at a lower wave number compared to a non-conjugated carbonyl. The ${ }^{1} \mathrm{H}-\mathrm{NMR}\left(\mathrm{CDCl}_{3}\right)$ spectra of these compounds exhibited the expected multiplet near $\delta 7.10-8.50 \mathrm{ppm}$ due to the presence of aromatic protons. The two different N-H protons appeared as two broad singlets at $\delta 9.07-9.58 \mathrm{ppm}$ and $\delta 10.66-12.81 \mathrm{ppm}$ respectively, whereas the pyrazole methyl protons showed up near $\delta 2.50 \mathrm{ppm}$ as a singlet. The characteristic chemical shifts for $\mathrm{F} / \mathrm{CF}_{3}$ were also detected in the ${ }^{19} \mathrm{~F}-\mathrm{NMR}\left(\mathrm{CDCl}_{3}\right)$ spectra of $\mathbf{6 a}-\mathbf{p}$.

\subsection{Antifungal Activity Bioassay}

The in vitro antifungal screening data of the pyrazole acyl thiourea derivatives are provided in Table 2. It was observed that these synthesized compounds showed weak to good antifungal activities against the tested fungi at $100 \mu \mathrm{g} / \mathrm{mL}$. Compounds $\mathbf{6 b}, \mathbf{6 h}$ and $\mathbf{6 l}$ were shown to inhibit the growth of G. zeae at $24.7 \%, 48.6 \%$, and $22.9 \%$, respectively; compounds $\mathbf{6 b}$ and $\mathbf{6 h}$ exhibited good activities on F. oxysporum at $50.3 \%$ and $57.9 \%$, respectively, while compounds $\mathbf{6 b}$ and $\mathbf{6 h}$ inhibited the growth of C. mandshurica at $48.7 \%$ and $57.9 \%$, respectively. These figures were slightly lower than those of hymexazol. Amongst the new products compound $\mathbf{6 h}$ exhibited similar activities as hymexozole on the corresponding fungi. Although, a definite structure activity relationship could not be established with the limited experimental data and available compounds, it appears that with the incorporation of a 2- $\mathrm{FC}_{6} \mathrm{H}_{4} \mathrm{CH}_{2} \mathrm{CH}_{2}$ - unit through $\mathrm{R}_{2}$ into thiourea in the resulting products 6 might have a positive influence, enhancing the antifungal activity of the designed compounds, From the activity differences between $\mathbf{6 h}$ and $\mathbf{6 i}$, we can conclude that different fluorine atom $(\mathrm{F})$ positions on benzene can result in different activity: when F was substituted at the 2-position of benzene (6h), it showed much higher activity than that of $\mathbf{6} \mathbf{i}$ (where $\mathrm{F}$ was substituted at the 3-position of benzene), this may be caused by a steric effect on the internal hydrogen bond.

Table 2. Inhibition effects of pyrazole acyl thiourea derivatives 6 on phytopathogenic fungi.

\begin{tabular}{cccc}
\hline Compd. (100 $\boldsymbol{\mu g} / \mathbf{m L})$ & G. zeae & F. oxysporum & C. mandshurica \\
\hline $\mathbf{6 a}$ & 2.84 & 1.46 & -5.79 \\
$\mathbf{6 b}$ & 24.7 & 50.3 & 48.7 \\
$\mathbf{6 c}$ & 8.24 & 6.92 & 1.98 \\
$\mathbf{6 d}$ & 12.8 & -3.21 & 8.16 \\
$\mathbf{6 e}$ & 9.57 & -1.15 & 8.47 \\
$\mathbf{6 f}$ & -0.57 & 4.39 & 3.42 \\
$\mathbf{6 g}$ & 17.6 & -1.46 & -3.68 \\
$\mathbf{6 h}$ & 48.6 & 57.9 & 59.7 \\
$\mathbf{6 i}$ & 19.9 & 4.09 & 6.57 \\
$\mathbf{6 j}$ & 4.55 & -3.80 & -6.31 \\
$\mathbf{6}$ & 15.4 & 11.8 & 7.91 \\
$\mathbf{6}$ & 22.9 & 0.86 & 4.80 \\
$\mathbf{6 m}$ & -1.14 & 6.73 & 3.42 \\
$\mathbf{6 n}$ & 5.85 & -0.29 & 5.08 \\
$\mathbf{6 0}$ & 7.18 & 5.19 & 11.9 \\
$\mathbf{6 p}$ & 3.46 & 9.22 & 4.24 \\
hymexazol & 69.7 & 67.1 & 69.5 \\
\hline
\end{tabular}




\subsection{Antiviral Activity Bioassay}

The antiviral activities of compounds $\mathbf{6 a}-\mathbf{p}$ against TMV were assayed by the reported method [20]. The results of the in vivo bioassay against TMV are given in Table 3. Ningnanmycin was used as a reference antiviral agent. The data provided in Table 3 indicate that the title compounds $\mathbf{6 a}-\mathbf{p}$ showed curative rates ranging from $19.76 \%-41.23 \%$.

Table 3. The curative effect of the compounds $\mathbf{6 a}-\mathbf{p}$ against TMV in vivo.

\begin{tabular}{ccc}
\hline Agent & Concentration $(\boldsymbol{\mu g} / \mathbf{m L})$ & Curative effect $\mathbf{( \% )}$ \\
\hline $\mathbf{6 a}$ & 500 & 41.23 \\
$\mathbf{6 b}$ & 500 & 29.74 \\
$\mathbf{6 c}$ & 500 & 35.17 \\
$\mathbf{6 d}$ & 500 & 25.47 \\
$\mathbf{6 e}$ & 500 & 35.44 \\
$\mathbf{6 f}$ & 500 & 37.95 \\
$\mathbf{6 g}$ & 500 & 32.71 \\
$\mathbf{6 h}$ & 500 & 28.07 \\
$\mathbf{6 i}$ & 500 & 23.45 \\
$\mathbf{6 j}$ & 500 & 19.76 \\
$\mathbf{6 k}$ & 500 & 27.84 \\
$\mathbf{6 0}$ & 500 & 31.82 \\
$\mathbf{6 m}$ & 500 & 32.39 \\
$\mathbf{6 n}$ & 500 & 30.09 \\
$\mathbf{6 0}$ & 500 & 22.07 \\
$\mathbf{6 p}$ & 500 & 27.08 \\
Ningnanmycin & 500 & 51.92 \\
\hline
\end{tabular}

\section{Experimental}

\subsection{General}

Unless otherwise stated, all the reagents and reactants were purchased from commercial suppliers. Melting points were uncorrected and were determined on a XT-4 binocular microscope (Beijing Tech Instrument Co., Beijing, China). The ${ }^{1} \mathrm{H}-\mathrm{NMR}$ and ${ }^{13} \mathrm{C}-\mathrm{NMR}$ spectra were recorded on a JEOL ECX $500 \mathrm{NMR}$ spectrometer (Tokyo, Japan) at room temperature operating at $500 \mathrm{MHz}$ for ${ }^{1} \mathrm{H}-\mathrm{NMR}$, $125 \mathrm{MHz}$ for ${ }^{13} \mathrm{C}-\mathrm{NMR}$, and $470 \mathrm{MHz}$ for ${ }^{19} \mathrm{~F}-\mathrm{NMR}$, using $\mathrm{CDCl}_{3}$ as solvents and TMS as an internal standard; infrared spectra were recorded in $\mathrm{KBr}$ on a Bruker VECTOR 22 spectrometer (Ettlingen, Germany); elemental analysis was performed on an Elemental Vario-III CHN analyzer (Hanau, Germany). The course of the reactions was monitored by TLC; analytical TLC was performed on silica gel $\mathrm{GF}_{254}$; column chromatographic purification was carried out using silica gel. The intermediates 1-3 were prepared according to the literature procedure [18]. 


\subsection{Preparation of the Intermediate Acyl Isothiocyanates $\mathbf{5}$}

The intermediates $3(30 \mathrm{mmol})$ and thionyl chloride $(15 \mathrm{~mL})$ were placed in a dried round-bottomed flask containing a magnetic stirrer bar. The reaction mixture was stirred at $80^{\circ} \mathrm{C}$ for $4 \mathrm{~h}$ until evolution of gas completely ceased. The excess thionyl chloride was carefully removed by rotary distillation to give a white solid to which were added ammonium thiocyanate $(36 \mathrm{mmol})$ in acetonitrile $(60 \mathrm{~mL})$ and six drops of PEG-400. The solution was stirred at room temperature for $3 \mathrm{~h}$, filtered and the solvent was removed on a rotary evaporator to afford a crude yellow solid which was further purified by column chromatography using a mixture of petroleum ether and ethyl acetate $(\mathrm{v} / \mathrm{v}=2: 1)$ as developing solvent to give pure acyl isothiocyanate 5. The appearance, melting points and yields of the intermediates $\mathbf{5 a}, \mathbf{5 b}$ are provided in Table 4.

Table 4. Appearance, melting points and yields of intermediates $\mathbf{5 a}-\mathbf{b}$.

\begin{tabular}{ccccc}
\hline Intermediate & $\mathbf{R}_{\mathbf{1}}$ & Appearance & m.p. $/{ }^{\circ} \mathbf{C}$ & Yield $/ \%$ \\
\hline $\mathbf{5 a}$ & $\mathrm{CH}_{3}$ & Yellow solid & $51 \sim 52{ }^{\circ} \mathrm{C}$ & $82.6 \%$ \\
$\mathbf{5 b}$ & $\mathrm{C}_{6} \mathrm{H}_{5}$ & White solid & $69 \sim 71{ }^{\circ} \mathrm{C}$ & $81.7 \%$ \\
\hline
\end{tabular}

\subsection{Preparation of Title Compounds $\mathbf{6 a}-\mathbf{p}$}

To a well stirred solution of acyl isothiocyanate $5(1.00 \mathrm{mmol})$ in THF $(15 \mathrm{~mL})$, a suitable fluorinated aromatic amine $(1.20 \mathrm{mmol})$ was added. The mixture was stirred at room temperature for $4 \mathrm{~h}$ and monitored by TLC. After completion of the reaction, the solid was filtered; the solvent was evaporated and the crude product was purified by preparative TLC with a mixture of petroleum ether and ethyl acetate $(\mathrm{v} / \mathrm{v}=3 / 1-1 / 3)$ to give title compounds $\mathbf{6 a}-\mathbf{p}$.

1-(2-Bromo-5-fluorophenyl)-3-(5-chloro-1,3-dimethyl-1H-pyrazole-4-carbonyl)thiourea (6a). White solid, yield 73.9\%; m.p. 148-150 ${ }^{\circ} \mathrm{C}$; ${ }^{1} \mathrm{H}-\mathrm{NMR}\left(\mathrm{CDCl}_{3}\right): \delta 12.65(1 \mathrm{H}$, brs, CSNH), $9.28(1 \mathrm{H}$, brs, CONHCS), 8.33 6.88 (3H, m, Ph-H), $3.85\left(3 \mathrm{H}, \mathrm{s}, \mathrm{CH}_{3}\right), 2.50\left(3 \mathrm{H}, \mathrm{s}, \mathrm{CH}_{3}\right) ;{ }^{13} \mathrm{C}-\mathrm{NMR}\left(\mathrm{CDCl}_{3}\right): \delta$ 178.6, 160.9, 152.3, 137.7, 133.5, 127,6, 115.1, 114.9, 113.8, 113.6, 112.1, 108.7, 36.7, 14.8; ${ }^{19}$ F-NMR $\left(\mathrm{CDCl}_{3}\right): \delta-116.64$; IR: $v$ 3396.6, $3145.9(\mathrm{~N}-\mathrm{H}), 1678.1(\mathrm{C}=\mathrm{O}), 1159.2(\mathrm{C}=\mathrm{S}) \mathrm{cm}^{-1}$; Anal. Calc. for $\mathrm{C}_{13} \mathrm{H}_{11} \mathrm{BrClFN}_{4} \mathrm{OS}$ : C 36.85\%, H 2.38\%, N 13.22\%. Found: C 37.00\%, H 2.14\%, N 13.38\%.

\section{1-(2-Bromo-4,6-difluorophenyl)-3-(5-chloro-1,3-dimethyl-1H-pyrazole-4-carbonyl)thiourea}

(6b).

White solid, yield 74.1\%; m.p. 84-86 ${ }^{\circ} \mathrm{C}$; ${ }^{1} \mathrm{H}-\mathrm{NMR}\left(\mathrm{CDCl}_{3}\right): \delta 11.74(1 \mathrm{H}$, brs, CSNH), $9.46(1 \mathrm{H}$, brs, CONHCS), 7.25 6.96 (2H, m, Ph-H), $3.84\left(3 \mathrm{H}, \mathrm{s}, \mathrm{CH}_{3}\right), 2.45\left(3 \mathrm{H}, \mathrm{s}, \mathrm{CH}_{3}\right) ;{ }^{13} \mathrm{C}-\mathrm{NMR}\left(\mathrm{CDCl}_{3}\right): \delta$ 181.3, 161.3, 152.1, 127.7, 124.0, 123.9, 122.8, 116.1, 115.9, 108.6, 104.7, 104.5, 104.3, 36.7, 14.7; ${ }^{19}$ F-NMR $\left(\mathrm{CDCl}_{3}\right): \delta-107.6,-109.1$; IR: $v$ 3338.1, $3095.8(\mathrm{~N}-\mathrm{H}), 1668.4(\mathrm{C}=\mathrm{O}), 1157.3(\mathrm{C}=\mathrm{S}) \mathrm{cm}^{-1}$; Anal. Calc. for $\mathrm{C}_{13} \mathrm{H}_{10} \mathrm{BrClF}_{2} \mathrm{~N}_{4} \mathrm{OS}$ : C 39.30\%, H 2.73\%, N 13.81\%. Found: C 39.30\%, H 2.54\%, N 13.98\%.

1-(4-Bromo-2-(trifluoromethyl)phenyl)-3-(5-chloro-1,3-dimethyl-1H-pyrazole-4-carbonyl)thiourea (6c). White solid, yield 71.9\%; m.p. $112-114{ }^{\circ} \mathrm{C} ;{ }^{1} \mathrm{H}-\mathrm{NMR}\left(\mathrm{CDCl}_{3}\right): \delta 12.43(1 \mathrm{H}$, brs, $\mathrm{CSNH}), 9.36(1 \mathrm{H}$, brs, CONHCS), 7.89 7.73 (3H, m, Ph-H), $3.86\left(3 \mathrm{H}, \mathrm{s}, \mathrm{CH}_{3}\right), 2.49\left(3 \mathrm{H}, \mathrm{s}, \mathrm{CH}_{3}\right) ;{ }^{13} \mathrm{C}-\mathrm{NMR}\left(\mathrm{CDCl}_{3}\right): \delta$ $180.7,161.3,152.4,135.3,134.7,131.4,129.4,127.6,127.0,126.8,123.5,121.3,120.8,108.7,36.7$, 
14.8; ${ }^{19}$ F-NMR $\left(\mathrm{CDCl}_{3}\right): \delta-61.36$; IR: $v$ 3373.5, $2993.5(\mathrm{~N}-\mathrm{H}), 1670.4(\mathrm{C}=\mathrm{O}), 1126.4(\mathrm{C}=\mathrm{S}) \mathrm{cm}^{-1}$;

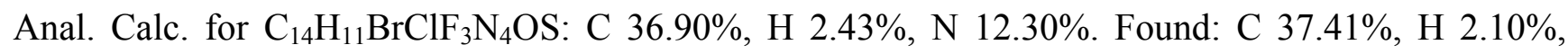
N 12.28\%.

1-(2-Fluorobenzyl)-3-(5-chloro-1,3-dimethyl-1H-pyrazole-4-carbonyl)thiourea (6d). White solid, yield 82.2\%; m.p. $121-122{ }^{\circ} \mathrm{C}$; ${ }^{1} \mathrm{H}-\mathrm{NMR}\left(\mathrm{CDCl}_{3}\right): \delta 10.92(1 \mathrm{H}$, brs, CSNH), $9.13(1 \mathrm{H}$, brs, CONHCS), 7.47 7.06 (4H, m, Ph-H), $4.96\left(2 \mathrm{H}, \mathrm{d}, J=5.75 \mathrm{~Hz} \mathrm{CH}_{2}\right), 3.83\left(3 \mathrm{H}, \mathrm{s}, \mathrm{CH}_{3}\right), 2.44(3 \mathrm{H}, \mathrm{s}$, $\left.\mathrm{CH}_{3}\right) ;{ }^{13} \mathrm{C}-\mathrm{NMR}\left(\mathrm{CDCl}_{3}\right): \delta 178.3,161.6,152.0,130.5,130.5,129.9,129.8,127.6,124.4,124.4$, 115.7, 115.5, 109.0, 43.5, 14.7; ${ }^{19} \mathrm{~F}-\mathrm{NMR}\left(\mathrm{CDCl}_{3}\right): \delta-117.88$; IR: $v$ 3398.6, $3228.8(\mathrm{~N}-\mathrm{H}), 1668.4$ $(\mathrm{C}=\mathrm{O}), 1166.9(\mathrm{C}=\mathrm{S}) \mathrm{cm}^{-1}$; Anal. Calc. for $\mathrm{C}_{14} \mathrm{H}_{14} \mathrm{ClFN}_{4} \mathrm{OS}: \mathrm{C} 49.34 \%, \mathrm{H} 4.14 \%, \mathrm{~N} 16.44 \%$. Found: C $49.57 \%$, H $3.93 \%$, N $16.93 \%$.

1-(2-Fluorophenyl)-3-(5-chloro-1,3-dimethyl-1H-pyrazole-4-carbonyl)thiourea (6e). White solid, yield 74.5\%; m.p. $152-154{ }^{\circ} \mathrm{C}$; ${ }^{1} \mathrm{H}-\mathrm{NMR}\left(\mathrm{CDCl}_{3}\right): \delta 12.56(1 \mathrm{H}$, brs, CSNH), $9.27(1 \mathrm{H}$, brs, CONHCS), 8.38 7.15 (4H, m, Ph-H), $3.87\left(3 \mathrm{H}, \mathrm{s}, \mathrm{CH}_{3}\right), 2.51\left(3 \mathrm{H}, \mathrm{s}, \mathrm{CH}_{3}\right) ;{ }^{13} \mathrm{C}-\mathrm{NMR}:\left(\mathrm{CDCl}_{3}, 125\right.$ $\mathrm{MHz}): \delta 178.8,161.3,152.3,127.8,127.7,127.6,126.0,125.6,124.0,115.7,115.6,108.9,36.7,14.8$; ${ }^{19} \mathrm{~F}-\mathrm{NMR}\left(\mathrm{CDCl}_{3}, 500 \mathrm{MHZ}\right): \delta-124.43$; IR $(\mathrm{KBr}): v 3383.1,2993.5(\mathrm{~N}-\mathrm{H}), 1666.5(\mathrm{C}=\mathrm{O}), 1141.9$ $(\mathrm{C}=\mathrm{S})$; Anal. Calcd for $\mathrm{C}_{13} \mathrm{H}_{12} \mathrm{ClFN}_{4} \mathrm{OS}$ : C 47.78\%, H 3.70\%, N 17.15\%; Found: C 47.45\%, H 3.83\%, N 17.21\%.

1-(2-Fluoro-5-(trifluoromethyl)phenyl)-3-(5-chloro-1,3-dimethyl-1H-pyrazole-4-carbonyl)thiourea (6f). White solid, yield 73.2\%; m.p. $123-125{ }^{\circ} \mathrm{C} ;{ }^{1} \mathrm{H}-\mathrm{NMR}\left(\mathrm{CDCl}_{3}\right): \delta 12.80(1 \mathrm{H}$, brs, $\mathrm{CSNH}), 9.30(1 \mathrm{H}$, brs, CONHCS), 8.95 7.27 (3H, m, Ph-H), $3.85\left(3 \mathrm{H}, \mathrm{s}, \mathrm{CH}_{3}\right), 2.49\left(3 \mathrm{H}, \mathrm{s}, \mathrm{CH}_{3}\right) ;{ }^{13} \mathrm{C}-\mathrm{NMR}:\left(\mathrm{CDCl}_{3}\right): \delta$ 178.7, 161.4, 152.3, 127.7, 126.9, 126.8, 126.7, 126.4, 124.5, 124.3, 122.3, 116.1, 116.0, 108.6, 36.6, 14.7; ${ }^{19} \mathrm{~F}-\mathrm{NMR}\left(\mathrm{CDCl}_{3}\right): \delta-61.1,-126.5$; IR $(\mathrm{KBr}): v 3373.5,3145.9(\mathrm{~N}-\mathrm{H}), 1670.3(\mathrm{C}=\mathrm{O}), 1163.1$ $(\mathrm{C}=\mathrm{S})$; Anal. Calcd for $\mathrm{C}_{14} \mathrm{H}_{11} \mathrm{ClF}_{4} \mathrm{~N}_{4} \mathrm{OS}$ : C $42.59 \%, \mathrm{H} 2.81 \%, \mathrm{~N} \mathrm{14.19 \%}$; Found: $\mathrm{C} 42.76 \%$, H $2.58 \%$, N $14.34 \%$.

1-(2-Fluoro-3-(trifluoromethyl)phenyl)-3-(5-chloro-1,3-dimethyl-1H-pyrazole-4-carbonyl)thiourea (6g). White solid, yield 74.9\%; m.p. $139-141{ }^{\circ} \mathrm{C} ;{ }^{1} \mathrm{H}-\mathrm{NMR}\left(\mathrm{CDCl}_{3}\right): \delta 12.80(1 \mathrm{H}$, brs, CSNH$), 9.31(1 \mathrm{H}$, brs, CONHCS), 8.75 7.26 (3H, m, Ph-H), $3.87\left(3 \mathrm{H}, \mathrm{s}, \mathrm{CH}_{3}\right), 2.51\left(3 \mathrm{H}, \mathrm{s}, \mathrm{CH}_{3}\right) ;{ }^{13} \mathrm{C}-\mathrm{NMR}:\left(\mathrm{CDCl}_{3}\right): \delta$ 180.1, 161.1, 151.8, 140.8, 140.8, 130.2, 130.1, 127.4, 124.4, 115.7, 115.6, 113.7, 113.5, 109.0, 36.7, 14.6; ${ }^{19} \mathrm{~F}-\mathrm{NMR}\left(\mathrm{CDCl}_{3}\right): \delta-62.0,-119.5$; IR $(\mathrm{KBr}): v 3387.0,3145.9(\mathrm{~N}-\mathrm{H}), 1680.0(\mathrm{C}=\mathrm{O}), 1143.8$ $(\mathrm{C}=\mathrm{S})$; Anal. Calcd for $\mathrm{C}_{13} \mathrm{H}_{11} \mathrm{BrClFN}_{4} \mathrm{OS}$ : C 42.59\%, H 2.81\%, N 14.19\%; Found: C 42.70\%, H $2.61 \%$, N $14.29 \%$.

1-(2-Fluorophenethyl)-3-(5-chloro-1,3-dimethyl-1H-pyrazole-4-carbonyl)thiourea (6h). White solid, yield 79.3\%; m.p. 97-99 ${ }^{\circ} \mathrm{C}$; ${ }^{1} \mathrm{H}-\mathrm{NMR}\left(\mathrm{CDCl}_{3}\right): \delta 10.66$ (1H, brs, CSNH), 9.07 (1H, brs, CONHCS), 7.28 7.00 (4H, m, Ph-H), 3.92 3.89 (2H, q, $\left.\mathrm{CH}_{2}\right), 3.79\left(3 \mathrm{H}, \mathrm{s}, \mathrm{CH}_{3}\right), 3.06\left(2 \mathrm{H}, \mathrm{t}, J=7.45 \mathrm{~Hz}, \mathrm{CH}_{2}\right)$, $2.40\left(3 \mathrm{H}, \mathrm{s}, \mathrm{CH}_{3}\right) ;{ }^{13} \mathrm{C}-\mathrm{NMR}:\left(\mathrm{CDCl}_{3}\right): \delta 180.0,161.0,151.6,131.1,128.5,127.4,125.2,125.1,124.3$, 115.4, 115.3, 108.9, 45.5, 36.6, 27.8, 14.6; ${ }^{19} \mathrm{~F}-\mathrm{NMR}\left(\mathrm{CDCl}_{3}\right): \delta-118.20$; IR (KBr): v 3385.1, 3244.3 $(\mathrm{N}-\mathrm{H}), 1666.5(\mathrm{C}=\mathrm{O}), 1159.2(\mathrm{C}=\mathrm{S})$; Anal. Calcd for $\mathrm{C}_{15} \mathrm{H}_{16} \mathrm{ClFN}_{4} \mathrm{OS}: \mathrm{C} \quad 50.77 \%, \mathrm{H} 4.55 \%$, N 15.79\%; Found: C 50.90\%, H 4.16\%, N 15.79\%. 
1-(3-Fluorophenethyl)-3-(5-chloro-1,3-dimethyl-1H-pyrazole-4-carbonyl)thiourea (6i). White solid, yield 78.8\%; m.p. $117-119{ }^{\circ} \mathrm{C} ;{ }^{1} \mathrm{H}-\mathrm{NMR}\left(\mathrm{CDCl}_{3}\right): \delta 10.68(1 \mathrm{H}$, brs, $\mathrm{CSNH}), 9.08(1 \mathrm{H}$, brs, CONHCS), 7.30 6.91 (4H, m, Ph-H), 3.93 3.89 (2H, q, $\left.\mathrm{CH}_{2}\right), 3.82\left(3 \mathrm{H}, \mathrm{s}, \mathrm{CH}_{3}\right), 3.03\left(2 \mathrm{H}, \mathrm{t}, J=8.00 \mathrm{~Hz}, \mathrm{CH}_{2}\right), 2.43$ $\left(3 \mathrm{H}, \mathrm{s}, \mathrm{CH}_{3}\right) ;{ }^{13} \mathrm{C}-\mathrm{NMR}:\left(\mathrm{CDCl}_{3}\right): \delta 180.1,161.1,151.8,140.8,130.2,127.4,124.4,115.7,115.6$, 113.7, 113.5, 109.0, 46.6, 36.6, 34.1, 14.6; ${ }^{19} \mathrm{~F}-\mathrm{NMR}\left(\mathrm{CDCl}_{3}\right): \delta$-112.97; IR (KBr): $v$ 3377.3, 3228.8 $(\mathrm{N}-\mathrm{H}), 1672.3(\mathrm{C}=\mathrm{O}), 1168.9(\mathrm{C}=\mathrm{S})$; Anal. Calcd for $\mathrm{C}_{15} \mathrm{H}_{16} \mathrm{ClFN}_{4} \mathrm{OS}: \mathrm{C} \quad 50.77 \%, \mathrm{H} 4.55 \%$, N $15.79 \%$; Found: C 50.55\%, H $4.21 \%$, N $15.99 \%$.

1-(5-Chloro-2-fluorophenyl)-3-(5-chloro-1,3-dimethyl-1H-pyrazole-4-carbonyl)thiourea (6j). White solid, yield 72.1\%; m.p. 158-160 ${ }^{\circ} \mathrm{C}$; ${ }^{1} \mathrm{H}-\mathrm{NMR}\left(\mathrm{CDCl}_{3}\right): \delta 12.56(1 \mathrm{H}$, brs, CSNH), $9.27(1 \mathrm{H}$, brs, CONHCS), 8.38 7.15 (4H, m, Ph-H), $3.87\left(3 \mathrm{H}, \mathrm{s}, \mathrm{CH}_{3}\right), 2.51\left(3 \mathrm{H}, \mathrm{s}, \mathrm{CH}_{3}\right) ;{ }^{13} \mathrm{C}-\mathrm{NMR}:\left(\mathrm{CDCl}_{3}\right): \delta$ $179.0,161.5,152.3,134.2,127.7,126.5,124.2,121.3,121.1,116.7,116.6,108.7,36.7,14.8$; ${ }^{19} \mathrm{~F}-\mathrm{NMR}\left(\mathrm{CDCl}_{3}\right): \delta-116.72$; IR $(\mathrm{KBr}): v 3375.4,2991.6(\mathrm{~N}-\mathrm{H}), 1670.4(\mathrm{C}=\mathrm{O}), 1138.0(\mathrm{C}=\mathrm{S})$; Anal. Calcd for $\mathrm{C}_{13} \mathrm{H}_{11} \mathrm{Cl}_{2} \mathrm{FN}_{4} \mathrm{OS}$ : C 43.23\%, H 3.07\%, N 15.51\%; Found: C 43.61\%, H 2.96\%, N 15.61\%.

1-(4-(Trifluoromethylthio)phenyl)-3-(5-chloro-1,3-dimethyl-1H-pyrazole-4-carbonyl)thiourea

(6k). White solid, yield 74.4\%; m.p. $145-147{ }^{\circ} \mathrm{C}$; ${ }^{1} \mathrm{H}-\mathrm{NMR}\left(\mathrm{CDCl}_{3}\right): \delta 12.75(1 \mathrm{H}$, brs, $\mathrm{CSNH}), 9.21(1 \mathrm{H}$, brs, CONHCS), 7.92 7.52 (4H, m, Ph-H), $3.86\left(3 \mathrm{H}, \mathrm{s}, \mathrm{CH}_{3}\right), 2.49\left(3 \mathrm{H}, \mathrm{s}, \mathrm{CH}_{3}\right) ;{ }^{13} \mathrm{C}-\mathrm{NMR}:\left(\mathrm{CDCl}_{3}\right)$ : $\delta$ 178.2, 161.5, 152.3, 140.2, 137.0, 133.3, 127.7, 124.2, 121.6, 108.8, 36.7, 14.8; ${ }^{19} \mathrm{FNMR}\left(\mathrm{CDCl}_{3}\right)$ : $\delta$-42.66; IR (KBr): v 3410.2, $3032.1(\mathrm{~N}-\mathrm{H}), 1666.5(\mathrm{C}=\mathrm{O}), 1139.9(\mathrm{C}=\mathrm{S})$; Anal. Calcd for $\mathrm{C}_{14} \mathrm{H}_{12} \mathrm{ClF}_{3} \mathrm{~N}_{4} \mathrm{OS}_{2}$ : C 41.13, H 2.96, N 13.70; Found: C 41.22, H 2.76, N 13.73.

1-(4-Bromo-2-(trifluoromethyl)phenyl)-3-(5-chloro-3-methyl-1-phenyl-1H-pyrazole-4-carbonyl) thiourea (6l): White solid, yield 75.9\%; m.p. $142-144{ }^{\circ} \mathrm{C} ;{ }^{1} \mathrm{H}-\mathrm{NMR}\left(\mathrm{CDCl}_{3}\right): \delta 12.43(1 \mathrm{H}$, brs, $\mathrm{CSNH}), 9.48$ (1H, brs, CONHCS), 7.89 7.52 (8H, m, Ph-H), $2.60\left(3 \mathrm{H}, \mathrm{s}, \mathrm{CH}_{3}\right) ;{ }^{13} \mathrm{C}-\mathrm{NMR}\left(\mathrm{CDCl}_{3}\right): \delta 180.7,161.5$, $153.5,136.9,135.4,134.7,131.4,129.7,129.5,127.4,127.2,126.9,125.6,120.9,110.2,15.0$; ${ }^{19} \mathrm{~F}-\mathrm{NMR}\left(\mathrm{CDCl}_{3}\right): \delta-61.39$; IR (KBr): $v$ 3373.5, $3119.2(\mathrm{~N}-\mathrm{H}), 1670.4(\mathrm{C}=\mathrm{O}), 1149.6(\mathrm{C}=\mathrm{S})$; Anal.

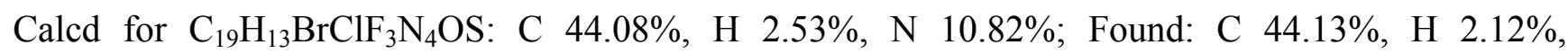
N 10.72\%.

1-(2-Fluorobenzyl)-3-(5-chloro-3-methyl-1-phenyl-1H-pyrazole-4-carbonyl)thiourea (6m). White solid, yield 82.9\%; m.p. 106-108 ${ }^{\circ} \mathrm{C}$; ${ }^{1} \mathrm{H}-\mathrm{NMR}\left(\mathrm{CDCl}_{3}\right): \delta 10.93(1 \mathrm{H}$, brs, CSNH), $9.27(1 \mathrm{H}$, brs, CONHCS), 7.54 7.07 (9H, m, Ph-H), $4.97\left(2 \mathrm{H}, \mathrm{d}, J=5.75 \mathrm{~Hz}, \mathrm{CH}_{2}\right), 2.53\left(3 \mathrm{H}, \mathrm{s}, \mathrm{CH}_{3}\right) ;{ }^{13} \mathrm{C}-\mathrm{NMR}$ $\left(\mathrm{CDCl}_{3}\right): \delta 180.1,161.2,152.9,136.9,130.5,130.5,129.9,129.6,129.4,125.5,124.4,124.4,115.7$, 115.6, 110.6, 43.5, 14.8; ${ }^{19} \mathrm{~F}-\mathrm{NMR}\left(\mathrm{CDCl}_{3}\right): \delta-117.80$; IR $(\mathrm{KBr}): v$ 3394.7, $3248.1(\mathrm{~N}-\mathrm{H}), 1666.5$ $(\mathrm{C}=\mathrm{O}), 1165.0(\mathrm{C}=\mathrm{S})$; Anal. Calcd for $\mathrm{C}_{19} \mathrm{H}_{16} \mathrm{ClFN}_{4} \mathrm{OS}$ : C 56.64\%, H 4.00\%, N 13.91\%; Found: C $57.07 \%$, H $4.46 \%$, N $14.22 \%$.

1-(2-Fluoro-5-(trifluoromethyl)phenyl)-3-(5-chloro-3-methyl-1-phenyl-1H-pyrazole-4-carbonyl) thiourea (6n). White solid, yield 75.1\%; m.p. $178-180{ }^{\circ} \mathrm{C} ;{ }^{1} \mathrm{H}-\mathrm{NMR}\left(\mathrm{CDCl}_{3}\right): \delta 12.77(1 \mathrm{H}$, brs, $\mathrm{CSNH}), 9.35$ (1H, brs, CONHCS), 7.92 7.52 (9H, m, Ph-H), $2.60\left(3 \mathrm{H}, \mathrm{s}, \mathrm{CH}_{3}\right) ;{ }^{13} \mathrm{C}-\mathrm{NMR}\left(\mathrm{CDCl}_{3}\right): \delta 178.7,161.5$, $153.4,136.9,129.7,129.5,127.4,126.8,126.7,125.6,124.5,122.6,116.2,116.1,110.2,15.0$; ${ }^{19} \mathrm{~F}-\mathrm{NMR}\left(\mathrm{CDCl}_{3}\right): \delta-61.98,-119.33$; IR $(\mathrm{KBr}): v$ 3215.3, $3076.5(\mathrm{~N}-\mathrm{H}), 1685.8(\mathrm{C}=\mathrm{O}), 1157.3$ 
(C=S); Anal. Calcd for $\mathrm{C}_{19} \mathrm{H}_{13} \mathrm{ClF}_{4} \mathrm{~N}_{4} \mathrm{OS}$ : C 49.95\%, $\mathrm{H} 2.87 \%$, N 12.26\%; Found: C 50.07\%, H $2.73 \%$, N $12.20 \%$.

1-(2-Fluoro-3-(trifluoromethyl)phenyl)-3-(5-chloro-3-methyl-1-phenyl-1H-pyrazole-4-carbonyl) thiourea (6o). White solid, yield 76.2\%; m.p. $146-148{ }^{\circ} \mathrm{C} ;{ }^{1} \mathrm{H}-\mathrm{NMR}\left(\mathrm{CDCl}_{3}\right): \delta 12.81$ (1H, brs, CSNH), 9.43 (1H, brs, CONHCS), 7.55 7.26 (8H, m, Ph-H), $2.61\left(3 \mathrm{H}, \mathrm{s}, \mathrm{CH}_{3}\right) ;{ }^{13} \mathrm{C}-\mathrm{NMR}\left(\mathrm{CDCl}_{3}\right): \delta 178.9,161.5$, $153.5,136.9,129.7,129.5,127.5,125.5,123.2,117.5,117.3,110.2,14.9 ;{ }^{19} \mathrm{~F}-\mathrm{NMR}\left(\mathrm{CDCl}_{3}\right): \delta$ -61.09, -126.50; IR (KBr): v 3221.7, $3086.2(\mathrm{~N}-\mathrm{H}), 1678.4(\mathrm{C}=\mathrm{O}), 1153.2(\mathrm{C}=\mathrm{S})$; Anal. Calcd for $\mathrm{C}_{19} \mathrm{H}_{13} \mathrm{ClF}_{4} \mathrm{~N}_{4} \mathrm{OS}$ : C 49.95\%, H 2.87\%, N 12.26\%; Found: C 49.91\%, H 2.77\%, N 12.31\%.

1-(4-(Trifluoromethylthio)phenyl)-3-(5-chloro-3-methyl-1-phenyl-1H-pyrazole-4-carbonyl)thiourea (6p). White solid, yield 75.7\%; m.p. ${ }^{109-111}{ }^{\circ} \mathrm{C}$; ${ }^{1} \mathrm{H}-\mathrm{NMR}\left(\mathrm{CDCl}_{3}\right): \delta 12.77(1 \mathrm{H}$, brs, $\mathrm{CSNH}), 9.35(1 \mathrm{H}$, brs, CONHCS), 7.92 7.52 (9H, m, Ph-H), $2.60\left(3 \mathrm{H}, \mathrm{s}, \mathrm{CH}_{3}\right) ;{ }^{13} \mathrm{C}-\mathrm{NMR}$ : $\left(\mathrm{CDCl}_{3}\right): \delta 178.1,161.6$, $153.2,140.2,137.0,136.9,129.7,129.5,127.4,125.5,124.1,121.8,110.2,15.0 ;{ }^{19} \mathrm{~F}-\mathrm{NMR}\left(\mathrm{CDCl}_{3}\right)$ : $\delta$-42.64; IR (KBr): $v$ 3388.9, $3032.1(\mathrm{~N}-\mathrm{H}), 1674.2(\mathrm{C}=\mathrm{O}), 1151.5(\mathrm{C}=\mathrm{S})$; Anal. Calcd for $\mathrm{C}_{19} \mathrm{H}_{14} \mathrm{ClF}_{3} \mathrm{~N}_{4} \mathrm{OS}_{2}$ : C 48.46\%, H 3.00\%, N $11.90 \%$; Found: C 48.36\%, H 2.97\%, N 11.87\%.

\subsection{Antifungal Bioassays}

The antifungal activity of all synthesized compounds was tested against F. oxysporum, G. zeae, and C. mandshurica by the poison plate technique [21]. All the compounds were dissolved in DMSO (10 $\mathrm{mL})$ before mixing with Potato Dextrose Agar (PDA, $90 \mathrm{~mL}$ ). The final concentration of the compounds in the medium was fixed at $100 \mu \mathrm{g} / \mathrm{mL}$. The three kinds of fungi were incubated in PDA at $25 \pm 1{ }^{\circ} \mathrm{C}$ for 5 days to get new mycelium for the antifungal assays, and then a mycelia disk of approximately $0.45 \mathrm{~cm}$ diameter cut from the culture medium was picked up with a sterilized inoculation needle and inoculated in the center of PDA plate. The inoculated plates were incubated at $25 \pm 1{ }^{\circ} \mathrm{C}$ for 5 days. DMSO in sterilized distilled water served as control, while hymexazole was used as positive control for each treatment with three replicates being conducted for each experiment. The radial growth of the fungal colonies was measured on the sixth day and the data were statistically analyzed. The in vitro inhibiting effects of the test compounds on the fungi were calculated by the formula $C V=(A-B) / A$, where A represents the diameter of fungi growth on untreated PDA, B represents the diameter of fungi on treated PDA, and CV represents the rate of inhibition.

\subsection{Antiviral Activity Bioassay}

Purification of tobacco mosaic virus. Using Gooding's method [22], the upper leaves of Nicotiana tabacum L. Inoculated with TMV were selected and ground in phosphate buffer, then filtered through double layer pledget. The filtrate was centrifuged at 10,000 g, treated twice with PEG and centrifuged again. The whole experiment was carried out $4{ }^{\circ} \mathrm{C}$. Absorbance values were estimated at $260 \mathrm{~nm}$ using an ultraviolet spectrophotometer:

$$
\text { virus concn }=\left(\mathrm{A}_{260} \times \text { dilution ratio }\right) / E^{1 \mathrm{~cm}}
$$


Curative effect of compounds against TMV in vivo. Growing leaves of Nicotiana tabacum. L. of the same ages were selected. The tobacco mosaic virus (concentration of $6 \times 10^{-3} \mathrm{mg} / \mathrm{mL}$ ) was dipped and inoculated on the whole leaves. Then the leaves were washed with water and dried. The compound solution was smeared on the left side and the solvent was on the right side for control. The local lesion numbers were then recorded 3-4 days after inoculation [23]. For each compound, three repetitions were conducted to ensure the reliability of the results, which were measured according to the following formula:

Inhibition rate $(\%)=\underline{\text { av local lesion numbers of control (not treated with compound) }- \text { av local lesion numbers smeared with drugs }}$ av local lesion numbers without drugs

\section{Conclusions}

In the present study, a mild and effective method for the preparation of sixteen novel pyrazole acyl thiourea derivatives employing monomethylhydrazine (phenylhydrazine) and ethyl acetoacetate as the starting materials is described. The synthesized compounds were characterized by spectral data $\left({ }^{1} \mathrm{H}-\mathrm{NMR},{ }^{13} \mathrm{C}-\mathrm{NMR},{ }^{19} \mathrm{~F}-\mathrm{NMR}, \mathrm{IR}\right)$ and elemental analysis. The compounds were subjected to in vitro fungicidal activity assays against $G$. zeae, F. oxysporum and $C$. mandshurica. The results showed that the synthesized pyridazine compounds possessed weak to good antifungal activities against the tested fungi, with compounds $\mathbf{6 b}, \mathbf{6} \mathbf{h}$ displaying good activity. Preliminary bioassays indicated that some of these compounds are also associated with good inhibitory activities against TMV at a concentration of $500 \mathrm{mg} / \mathrm{L}$. Further studies are currently underway to establish a definite structure activity relationship.

\section{Acknowledgements}

We are grateful to the National Natural Science Foundation of China (No. 2010CB126105) and the National Key Technologies R\&D Program of China (No. 2011BAE06B05-6) and the National Natural Science Foundation of China (No. 20962005) for supporting the project.

\section{References and Notes}

1. Duan, L.P.; Xue, J.; Xu, L.L.; Zhang, H.B. Synthesis 1-acyl-3-(2'-aminophenyl) thioureas as antiintestinal nematode prodrugs. Molecules 2010, 15, 6941-6947.

2. Zhong, Z.M.; Xing, R.; Liu, S.; Wang, L.; Cai, S.B.; Li, P.C. Synthesis of acyl thiourea derivatives of chitosan and their antimicrobial activities in vitro. Carbohydr. Res. 2008, 343, 566-570.

3. Wang, Z.Y.; Wang, S.; Song, X.J.; Wang, Y.G. Synthesis and biological activity of $\mathrm{N}$-5-(1-o-chlorophenoxyethy1)-1,3,4-thiodiazol-2-y1]-N-aryloxyacetyl thioureas. Chin. J. Pestic. Sci. 2005, 7, 282-284.

4. Jurasek, A.; Safer, P.; Zvak, V. Synthesis and reactions of $N$-aryl-N'-(3-furoyl) thioureas. Chem. Pap. 1987, 41, 693-702.

5. Nakamura, K.; Koike, K.; Sakamoto, M.; Nasumo, I. Pyrazole Derivatives. US 5607898, 1997; [Chem. Abstr. 1998, 125, 275866].

6. Dorfmeister, G.; Franke, H.; Geisler, J.; Hartfiel, U.; Bohner, J.; Rees, R. Substituted Pyrazole Derivatives and Their Use as Herbicides. US 5756424, 1998; [Chem. Abstr. 1998, 121, 230765]. 
7. Elbe, H.; Rieck, H.; Dunkel, R. Pyrazolyl Biphenyl Carboxamides. WO 02008195, 2002; [Chem. Abstr. 2002, 136, 151162].

8. Chen, H.S.; Li, Z.M.; Han, Y.F. Synthesis andfungicidal activity against Rhizoctonia solani of 2-alkyl(alkylthio)-5-pyrazoryl-1,3,4-oxadiazoles(thiodiazoles). J. Agric. Food Chem. 2002, 50, 3757-3760.

9. Xu, D.F.; Li, J.Z.; Guo, Y.L.; Xue, S.J. Synthesis and biological activity of $N^{\prime}$-(substituted pyrimidin-2-yl)- $N$-chrysanthemoylthiourea derivatives. Chin. J. Org. Chem. 2005, 25, 1053-1056.

10. Wang, Z.Y.; Gong, Y.X.; Chen, C.B.; Song, X.J.; Wang, Y.G. Synthesis and biological activity of $N$-[5-(1-o(p)-chlorophenoxy-ethyl)-1,3,4-thiodiazol-2-yl]- $N$ '-aroyl thioureas. Chin. J. Org. Chem. 2005, 25, 1306-1310.

11. Gong, Y.X.; Wang, Z.Y.; Zhang, Z.W.; Chen, C.B.; Wang, Y.G. Synthesis and biological activity of $N$-(2-carboxyl-1,3,4-thiadizol-5-yl)- $N$ '-aroyl thioureas and aryloxyacetyl thioureas. Chin. J. Org. Chem. 2006, 26, 360-363.

12. Wang, S.; Feng, G.R.; Liu, G.H.; Ye, W.F.; Wang, Y.G. Synthesis and biological activity of $N$-(1,2,4-triazolo-[1,5-a]pyrimidin-2-yl)-N'-aroyl thioureas. Chin. J. Org. Chem. 2006, 26, 1584-1586.

13. Wang, Q.D.; Xue, S.J.; Shen, J.F.; Cai, Z.J. Synthesis and bioactivity of $N$-[5-(2-chlorophenyl)-2furamidothiocarbonyl]- $L$ - $\alpha$-amino acid ethyl esters. Chin. J. Org. Chem. 2008, 28, 521-524.

14. Yan, M.; Shi, D.Q. Synthesis and herbicidal activity of $N$-(4,6-disubstituted pyrimidin-2-yl)- $N^{\prime}-$ [1-(heteroaryl)methyl-5-methyl-1H-1,2,3-triazol-4-ylcarbonyl]thiourea. Chin. J. Org. Chem. 2008, 28, 736-740.

15. Abd El-Wahab, A.H.F.; Al-Fifi, Z.I.A.; Bedair, A.H.; Ali, F.M.; Halawa, A.H.A.; El-Agrody, A.M. Synthesis, reactions and biological evaluation of some new naphtho[2,1-b]furan derivatives bearing a pyrazole nucleus. Molecules 2011, 16, 307-318.

16. Ouyang, G.P.; Cai, X.J.; Chen, Z.; Song, B.A.; Bhadury, P.S.; Yang, S.; Jin, L.H.; Xue, W.; $\mathrm{Hu}$, D.Y.; Zeng, S. Synthesis and antiviral activities of pyrazole derivatives containing oxime ethers moiety. J. Agric Food Chem. 2008, 56, 10160-10167.

17. Saad, H.A.; Osman, N.A.; Moustafa, A.H. Synthesis and analgesic activity of some new pyrazoles and triazoles bearing a 6,8-dibromo-2-methylquinazoline moiety. Molecules 2011, 16, 10187-10201.

18. Wei, T.B.; Zhang, Y.M.; Gao, L.M. An efficient synthesis of polymethylene-bis-aroyl thiourea derivatives under the condition of phase transfer catalysis. Synth. Commun. 2000, 30, 493-500.

19. Illi, V.O. Phase transfer catalyzed acylation. Tetrahedron Lett. 1979, 20, 2431-2432.

20. Lv, Y.P.; Wang, X.Y.; Song, B.A.; Yang, S.; Yan, K.; Xu, G.F.; Bhadury, P.S.; Liu, F.; Jin, L.H.; $\mathrm{Hu}, \mathrm{D} . Y$. Synthesis, antiviral and antifungal bioactivity of 2-cyanoacrylate derivatives containing phosphonyl moieties. Molecules 2007, 12, 965-978.

21. Ouyang, G.; Zhang, P.; Xu, G.; Song, B.; Yang, S.; Jin, L.; Xue, W.; Hu, D.; Lu, P.; Chen, Z. Synthesis and antifungal bioactivities of 3-alkylquinazolin-4-one derivatives. Molecules 2006, 11, 383-392.

22. Gooding, G.V., Jr.; Hebert, T.T. A simple technique for purification of tobacco mosaic virus in large quantities. Phytopathology 1967, 57, 1285-1290.

23. Gao, X.; Cai, X.; Yan, K.; Song, B.; Gao, L.; Chen, Z. Synthesis and antiviral bioactivities of 2-aryl- or 2-methyl-3-(substituted-Benzalamino)-4(3H)-quinazolinone derivatives. Molecules 2007, 12, 2621-2642. 
Sample Availability: Samples of the intermediates 1-5 and title compounds $\mathbf{6 a - p}$ are available from the authors.

(C) 2012 by the authors; licensee MDPI, Basel, Switzerland. This article is an open access article distributed under the terms and conditions of the Creative Commons Attribution license (http://creativecommons.org/licenses/by/3.0/). 